\title{
Powassan virus - an emerging public health concern
}

\author{
Chloe Bogaty MD, Michael Drebot PhD
}

Cite as: CMAJ 2018 April 16;190:E472. doi: 10.1503/cmaj.171305

1

A flu-like illness during the summer suggests a possible mosquitoor tick-borne infection

Investigation of a summertime flu-like illness is warranted. Well-known pathogens include Lyme disease, anaplasma and West Nile virus. A lesser-known pathogen is Powassan virus (POWV).

2 POWV is emerging as an important pathogen in some provinces owing to rapid spread of its vector

Powassan virus is transmitted by the blacklegged tick Ixodes scapularis. ${ }^{1}$ Ticks harbouring POWV have been detected in Nova Scotia, Ontario and Manitoba, with expansion of the tick across eastern and central Canada (Field Studies section, Zoonotic Diseases and Special Pathogens, National Microbiology Laboratory, Public Health Agency of Canada: unpublished data, 2017). This suggests a risk for increased transmission of POWV, especially in individuals who engage in outdoor work or recreational activities. ${ }^{2}$ Current rates of disease are not well documented, which may reflect underinvestigation.

\section{3}

\section{POWV can cause severe disease of the central nervous system}

The incubation period for POWV infection is one to five weeks and most cases remain asymptomatic. Manifestations of the illness include fever and flu-like symptoms, and, more uncommonly, meningoencephalitis, with findings of meningismus, altered mental status, seizures or cranial nerve palsies. Mortality is estimated at $5 \%$ to $10 \%$ in severe cases with neurologic involvement, and neurologic sequelae are common. ${ }^{3}$

\section{Serology is the principal diagnostic test for POWV}

Serology testing should be requested for patients with compatible symptoms and risk factors for tick exposure. Serology is offered at the National Microbiology Laboratory through provincial public health laboratories. Acute serologic samples are examined for POWV-specific immunoglobulin M in serum or cerebrospinal fluid, which usually provides a positive result two to three days after symptom onset. A positive result for immunoglobulin $G$ in serum is diagnostic if it shows seroconversion or a substantial rise in antibody titres in convalescent samples drawn two to three weeks later (Field Studies section, Zoonotic Diseases and Special Pathogens, National Microbiology Laboratory, Public Health Agency of Canada: unpublished data, 2017).

\section{Prevention of tick bites is the best defense}

There are no specific treatments or vaccines for POWV. Important measures to prevent tick bites include wearing protective clothing, using $N, N$-diethyl-m-toluamide (DEET) insect repellent, and performing thorough checks after outdoor activities and promptly removing ticks. ${ }^{4}$

\section{References}

1. Ebel GD. Update on Powassan virus: emergence of a North American tick-borne flavivirus. Annu Rev Entomol 2010;55:95-110.

2. Piantadosi A, Rubin DB, McQuillen DP, et al. Emerging cases of Powassan virus encephalitis in New England: clinical presentation, imaging, and review of the literature. Clin Infect Dis 2016;62:707-13.

3. Hinten SR, Beckett GA, Gensheimer KF, et al. Increased recognition of Powassan encephalitis in the United States, 1999-2005. Vector Borne Zoonotic Dis 2008;8: 733-40.

4. Ogden NH, Lindsay LR, Schofield SW. Methods to prevent tick bites and Lyme disease. Clin Lab Med 2015;35:883-99.

Competing interests: None declared.

This article has been peer reviewed.

Affiliations: Department of Medical Microbiology and Infectious Diseases (Bogaty), Max Rady College of Medicine, University of Manitoba; National Microbiology Laboratory (Drebot), Public Health Agency of Canada, Winnipeg, Man.

Acknowledgments: The authors thank Robbin Lindsay and Guillaume Poliquin for their thoughtful suggestions and reviews of this manuscript.

Correspondence to: Chloe Bogaty, chloe. bogaty@mail.mcgill.ca

CMAJ invites submissions to "Five things to know about ..." Submit manuscripts online at http://mc.manuscriptcentral.com/cmaj 